\title{
Is it Time to Change the Control Placebo Arms in Phase III Trials of Metastatic Castration Resistant Prostate Cancer?
}

\author{
Mutlu Dogan, Gokmen Umut Erdem*, Nurullah Zengin
}

\begin{abstract}
Prostate cancer is common all around the world. Hormonal therapy is the mainstay of therapy, however castration-resistant prostate cancer (CRPC) becomes a serious problem and needs further clinical trials with novel agents. Novel agents like cabazitaxel, abireterone acetate or enzalutamide are encouraging but we do not know which one is the best in metastatic CRPC. In here, treatment modalities for metastatic CRPC are discussed witha mini-review of the literature.
\end{abstract}

Keywords: Castration-resistant prostate cancer - abiraterone acetate - cabazitaxel - enzalutamide

Asian Pac J Cancer Prev, 16 (14), 6167-6169

\section{Introduction}

Prostate cancer is the most prevalent cancer in the male population in Western countries. According to recent evidence, it is the second leading cause of cancer-related death among men in the US (Iranikhah et al., 2014). The incidence increases in parallel to the increase in geriatric population.

Bone is the most common site for metastasis. Hormonal therapy is the main treatment modality with a median response duration of 12-33 months in metastatic prostate cancer, but most of them become castration resistant after a while (Lee et al., 2014). Docetaxel-based chemotherapy is the first choice of chemotherapy in metastatic castrationresistant prostate cancer (CRPC). However, treatment of those progressed under docetaxel-based chemotherapy led the investigators to use novel agents like cabazitaxel, abiraterone acetate or enzalutamide in metastatic CRPC (de Bono et al., 2010; 2011; Beer et al., 2014) (see Table 1).

Enzalutamide is an androgen receptor inhibitor which

Table 1. Phase III Trials of Metastatic Castration Resistant Prostate Cancer

\begin{tabular}{|c|c|c|c|c|}
\hline Trial Name & Study design/population Phase & $\begin{array}{l}\text { Drug dose Primary } \\
\text { Endpoint }\end{array}$ & Results & OS \\
\hline \multicolumn{5}{|c|}{ Published Results } \\
\hline $\begin{array}{l}\text { De Bono et } \\
\text { al, } 2010 \text {. }\end{array}$ & $\mathrm{R}, \mathrm{OL}, \mathrm{M}$ & $\begin{array}{l}\mathrm{C}+\mathrm{P}^{*}: \text { C } 25 \mathrm{mg} / \mathrm{m}^{2} \mathrm{q} \\
3 \mathrm{wk}+\mathrm{P}^{*} \\
\mathrm{MP}^{*}: \text { M } 12 \mathrm{mg} / \mathrm{m}^{2} \mathrm{q} \\
3 \mathrm{wk}+\mathrm{P}^{*}\end{array}$ & \multirow{5}{*}{$\begin{array}{l}\text { Median OS: } 12.7 \text { mo MP*, } 15.1 \mathrm{mo} \mathrm{CP} * \text {; } \\
\text { HR: } 0.7 ; \mathrm{p}<0.0001 ; 30 \% \text { reduction in the } \\
\text { risk of death } \\
\text { Median PFS: } 1.4 \mathrm{mo} \mathrm{MP}^{*}, 2.8 \mathrm{mo} \mathrm{CP}^{*} \text {; } \\
\text { HR: } 0.7 ; \text { p }<0.0001 \\
\text { Safety profile was predictable and } \\
\text { manageble }\end{array}$} & \multirow{5}{*}{$\begin{array}{l}2.4 \\
\text { mo }\end{array}$} \\
\hline $\begin{array}{l}\text { TROPIC } \\
\text { study }\end{array}$ & $\begin{array}{l}\text { Patients with mCRPC following docetaxel } \\
\text { therapy. }\end{array}$ & \multirow{4}{*}{ OS } & & \\
\hline & $\mathrm{M}+\mathrm{P}^{*}(\mathrm{n}: 377)$ & & & \\
\hline & $\mathrm{C}+\mathrm{P}^{*}(\mathrm{n}: 378)$ & & & \\
\hline & Phase III & & & \\
\hline \multirow[t]{4}{*}{$\begin{array}{l}\text { De Bono et } \\
\text { al., } 2011 \text {. }\end{array}$} & $\mathrm{R}(2: 1), \mathrm{DB}, \mathrm{M}, \mathrm{PC}$ & $\begin{array}{l}\text { A+P*: Abiraterone } 1 \\
\text { gr/d+ } \mathrm{P}^{*} \\
\mathrm{P}+\mathrm{P} \cdot *: \text { Placebo } \\
\text { daily+ }+\mathrm{P}^{*}\end{array}$ & \multirow[t]{4}{*}{$\begin{array}{l}\text { OS: } 14.8 \text { mo A+P* } 10.9 \text { mo P+P*; HR: } \\
0,65 ; \mathrm{p}<0.001\end{array}$} & \multirow{4}{*}{$\begin{array}{l}3.9 \\
\text { mo }\end{array}$} \\
\hline & $\begin{array}{l}\text { Failed } 1 \text { or } 2 \text { chemotherapy regimens, one of } \\
\text { which contained docetaxel }\end{array}$ & \multirow{3}{*}{ OS } & & \\
\hline & $\mathrm{A}+\mathrm{P}^{*}(\mathrm{n}: 797)$ & & & \\
\hline & Phase III & & & \\
\hline
\end{tabular}

Dept of Medical Oncology, Ankara Numune Training and Research Hospital, Altindag, Ankara, Turkey *For correspondence: gokmenumut@hotmail.com 
Table 1. (continued) Phase III Trials of Metastatic Castration Resistant Prostate Cancer

\begin{tabular}{|c|c|c|c|c|}
\hline \multirow{4}{*}{$\begin{array}{l}\text { Scher et al } \\
2012 \\
\text { AFFIRM }\end{array}$} & $\mathrm{R}(2: 1), \mathrm{DB}, \mathrm{M}, \mathrm{PC}$ & $\begin{array}{l}\text { Enzalutamide } 160 \\
\mathrm{mg} / \mathrm{d} \\
\text { Placebo }\end{array}$ & $\begin{array}{l}\text { Median OS: } 18.4 \text { mo E, } 13.6 \text { mo P; } \\
\text { HR:0.63; }<<0.001\end{array}$ & \multirow{4}{*}{$\begin{array}{l}4.8 \\
\text { mo }\end{array}$} \\
\hline & $\begin{array}{l}\text { Patients with mCRPC following docetaxel } \\
\text { therapy. }\end{array}$ & \multirow{3}{*}{ OS } & ORR: $29 \%$ E, $4 \%$ P & \\
\hline & $\begin{array}{l}\text { E (n: 800) } \\
\text { P (n:399) }\end{array}$ & & PSA PFS: $8.3 \mathrm{mo} \mathrm{E,} 3 \mathrm{mo} \mathrm{P}$ & \\
\hline & Phase III & & & \\
\hline \multirow[t]{2}{*}{$\begin{array}{l}\text { Beer et al } \\
\text { (Beer et al., } \\
\text { 2014). }\end{array}$} & $\mathrm{R}, \mathrm{DB}, \mathrm{OL}, \mathrm{M}, \mathrm{PC}$ & $\begin{array}{l}\text { E } 160 \mathrm{mg} / \mathrm{d} \\
\text { Placebo (no Pred- } \\
\text { nisone) }\end{array}$ & $\begin{array}{l}\text { rPFS: } 65 \% \text { E, } 14 \% \text { P } 81 \% \text { risk reduc- } \\
\text { tion; } \quad \text { HR: } 0.19 ; \mathrm{p}<0.001\end{array}$ & \multirow{4}{*}{$\begin{array}{r}2.2 \\
\text { mo } \\
\text { (est) }\end{array}$} \\
\hline & Pre chemo & \multirow{3}{*}{$\begin{array}{c}\text { rPFS } \\
\text { OS }\end{array}$} & $\begin{array}{l}\text { OS: } 29 \% \text { reduction in the risk of death; } \\
\text { HR: } 0.71 ; p<0.001\end{array}$ & \\
\hline \multirow{2}{*}{$\begin{array}{l}\text { PREVAIL } \\
\text { study } \\
\text { İnterim } \\
\text { analysis }\end{array}$} & $\begin{array}{l}E(n: 872) \\
P(n: 845)\end{array}$ & & $\begin{array}{l}\text { Median time until initiation of cytotoxic } \\
\text { chemotherapy: } 28 \text { mo E, } 10.8 \text { mo P }\end{array}$ & \\
\hline & Phase III & & OR: $59 \%$ E, $5 \%$ P; $p<0.0001$ & \\
\hline \multirow{4}{*}{$\begin{array}{l}\text { Lee at al } \\
\text { (Lee et al., } \\
\text { 2014). }\end{array}$} & $\begin{array}{l}\text { Patients with mCRPC following docetaxel } \\
\text { therapy. }\end{array}$ & $\begin{array}{l}\text { CP*: C } 25 \mathrm{mg} / \mathrm{m}^{2} \mathrm{q} 3 \\
\mathrm{wk}+\mathrm{P}^{*}\end{array}$ & PSA response: $32 \%$ & \\
\hline & $\mathrm{CP}^{*}(\mathrm{n}: 26)$ & \multirow{3}{*}{$\begin{array}{l}\text { Safety } \\
\text { Efficacy }\end{array}$} & Median time to treatment failure: $4.2 \mathrm{mo}$ & \\
\hline & & & Median time to progression: $8.5 \mathrm{mo}$ & \\
\hline & & & Median OS: $16.5 \mathrm{mo}$ & \\
\hline
\end{tabular}

AP*; abtaterone+prednisone, CP*; cabazitaxel+prednisone, d; day, DB; double-blind, E; enzalutamide, est; estimated, HR; hazard ratio, mCRPC; metastatic castration-resistant prostate cancer, mo; months, M; multicenter, MP*; mitoxantrone+ prednisone, PC; placebo-controled, PFS; progression-free survival, OL; open-label, OR; objective response, OS; overall survival, P; placebo, PP*; placebo+prednisone, R; randomized, rPFS; radiographic progression-free survival, wk; week; *Prednisone $5 \mathrm{mg}$ BID

was shown to have survival benefit in prostate cancer (Scher et al., 2012; Beer et al., 2014). Beer et al reported that enzalutamide has significantly decreased death rate
\& radiological progression in metastatic CRPC recently (Beer et al., 2014). The results are encouraging. However, enzalutamide was compared with placebo as a standard

Table 2. Ongoing Phase II Clinical Trials of Enzalutumab in Prostate Cancer

\begin{tabular}{|c|c|c|c|c|}
\hline Trial Name & Study design/population & Drug dose & $\begin{array}{l}\text { Primary End- } \\
\text { point }\end{array}$ & Comment \\
\hline \multicolumn{5}{|c|}{ Ongoing phase II studies with enzalutamide in prostat cancer } \\
\hline \multirow{4}{*}{ Efstathiou et al 2014} & $\mathrm{OL}$ & \multirow{4}{*}{$\begin{array}{l}\text { E } 160 \mathrm{mg} / \mathrm{d} \text { and } \\
\text { Abiraterone } 1 \mathrm{gr} / \\
\mathrm{d}+\mathrm{P}^{*}\end{array}$} & \multirow{4}{*}{$\begin{array}{l}\text { Safety Ef- } \\
\text { ficacy } \\
\text { PK } \\
\text { DDI }\end{array}$} & Active arm PSA RR \\
\hline & $\begin{array}{l}\text { Histologically/cytologically } \\
\text { confirmed CRPC Bone metasta- } \\
\text { ses Ongoing androgen depriva- } \\
\text { tion therapy }\end{array}$ & & & $\begin{array}{l}\text { Maximum PSA decline } \\
\geq 50 \%(37 / 49[76 \%]) \\
\geq 90 \%(22 / 49[45 \%]) \\
\text { PSA } \leq 0.1 \mathrm{ng} / \mathrm{ml}(5 / 49[10 \%])\end{array}$ \\
\hline & \multirow[t]{2}{*}{$\mathrm{E}+\mathrm{A}(\mathrm{n}: 60)$} & & & $\begin{array}{l}\text { E+ A combination has a favorable } \\
\text { safety profile, without clinically } \\
\text { meaningful PK DDI. }\end{array}$ \\
\hline & & & & $\begin{array}{l}11 / 60(18 \%) \text { patients progressed } \\
\text { within } 4 \text { months }\end{array}$ \\
\hline \multirow{3}{*}{ Alliance } & OL, R & E $160 \mathrm{mg} / \mathrm{d}$ & \multirow{3}{*}{ OS } & ClinicalTrials.gov Identifier: \\
\hline & Arm A: Enzalutamide & $\mathrm{E}+\mathrm{A}+\mathrm{P}: \mathrm{E}$ & & NCT01949337 \\
\hline & $\begin{array}{l}\text { Arm B: Emzalutamide+ Abi- } \\
\text { rateron+ Prednisone }(\mathrm{E}+\mathrm{A}+\mathrm{P})\end{array}$ & $\begin{array}{l}160 \mathrm{mg} / \mathrm{d} \text { and } \\
\text { Abiraterone } 1 \mathrm{gr} / \\
\mathrm{d}+\mathrm{P}^{*}\end{array}$ & & \\
\hline \multirow{3}{*}{$\begin{array}{l}\text { M.D. Anderson Cancer } \\
\text { Center }\end{array}$} & $\mathrm{R}, \mathrm{OL}$ & \multirow{3}{*}{$\begin{array}{l}\text { Abiraterone } 1 \\
\text { gr/d P*E } 160 \\
\text { mg/d LHRHa } \\
\text { monthly injec- } \\
\text { tion or three- } \\
\text { month injection }\end{array}$} & Safety & ClinicalTrials.gov Identifier: \\
\hline & $\begin{array}{l}\text { Arm A: Abiraterone Acetate + } \\
\text { Prednisone + Enzalutamide + } \\
\text { LHRHa }\end{array}$ & & \multirow[t]{2}{*}{ Efficacy } & \multirow[t]{2}{*}{ NCT01946165 } \\
\hline & $\begin{array}{l}\text { Arm B: Abiraterone Acetate + } \\
\text { Prednisone + LHRHa }\end{array}$ & & & \\
\hline
\end{tabular}

*CRPC; castration-resistant prostate cancer, DDI; drug-drug interactions, d; day, E; enzalutamide, E+A; enzalutamide+ abiraterone asetat, OL; open label, OS; overall survival, PK; pharmacokinetic, R; randomized, RR; response rate, LHRHa; luteinizing hormone-releasing hormone analog; *Prednisone 5 mg BID 
arm in this phase III trial. In this study, enzalutamide not only delayed initiation of subsequent chemotherapy but also led to a significant reduction in soft-tissue disease from baseline in $59 \%$ of the patients. It was well tolerated with similar rates of leaving study due to adverse events $(6 \%)$ in enzalutamide and placebo arms (Beer et al., 2014).

Cabazitaxel is a novel taxane which is effective even in metastatic taxane-resistant prostate cancer. Cabazitaxel had statistically and clinically significant overall survival benefit over mitoxantrone (de Bono et al., 2010). Abiraterone acetate was also shown to have activity in MPC by inhibiting androgen biosynthesis enzyme, CYP17. Increased survival was observed in all subgroups with a low rate of additional treatment-related toxicity in experimental arm (de Bono et al., 2011; Zhou et al., 2014).

In phase III trials of these novel agents, the standard arms were designed as mitoxantrone for cabazitaxel and placebo for both abiraterone acetate and enzalutamide (de Bono et al., 2010; de Bono et al., 2011; Beer et al., 2014). There was no statistically significant difference in overall survival of mCRPC patients in an indirect comparison of enzalutamide with abiraterone acetate (Tan et al., 2014). However, enzalutamide had significant benefits over abiraterone acetate for secondary outcomes, such as time to PSA progression, radiographic progression free survival and PSA response rates. Ongoing phase II clinical trials are listed in Table 2.

We consider that it is time to compare these novel agents with each other instead of placebo since they were shown to have statistically significant activity in previous phase III trials. So, it might contribute to the choice of optimal treatment for metastatic CRPC.

\section{Acknowledgements}

M.D., G. U. E and N.Z. have disclosed that they have no significant relationships with or financial interests in any commercial companies related to this study or article.

\section{References}

BeerTM, Armstrong AJ, Rathkopf DE, et al (2014). Enzalutamide in metastatic prostate cancer before chemotherapy. $N$ Engl J Med, 371, 424-33.

de Bono JS, Logothetis CJ, Molina A, et al (2011). Abiraterone and increased survival in metastatic prostate cancer. $N E n g l$ J Med, 364, 1995-2005.

de Bono JS, Oudard S, Ozguroglu M, et al (2010). Prednisone plus cabazitaxel or mitoxantrone for metastatic castrationresistant prostate cancer progressing after docetaxel treatment: a randomised open-label trial. Lancet, 376, 1147-54.

Efstathiou E, Titus MA, Wen S et al (2014) Enzalutamide (ENZA) in combination with abiraterone acetate (AA) in bone metastatic castration resistant prostate cancer (mCRPC). J Clin Oncol, 32, 5000.

Iranikhah M, Stricker S, Freeman MK (2014). Future of bisphosphonates and denosumab for men with advanced prostate cancer. Cancer Manag Res, 6, 217-24.

Lee JL, Park SH, Koh SJ, et al (2014). Effectiveness and safety of cabazitaxel plus prednisolone chemotherapy for metastatic castration-resistant prostatic carcinoma: data on Korean patients obtained by the cabazitaxel compassionate-use program. Cancer Chemother Pharmacol. [Epub ahead of print].

Scher HI, Fizazi K, Saad F, et al (2012). Increased survival with enzalutamide in prostate cancer after chemotherapy. $N$ Engl $J$ Med, 367, 1187-97.

Tan PS, Haaland B, Montero AJ, et al (2014). Hormonal therapeutics enzalutamide and abiraterone acetate in the treatment of metastatic castration-resistant prostate cancer (mCRPC) Post-docetaxel-an Indirect Comparison. Clin Med Insights Oncol, 8, 29-36.

Zhou ZR, Liu SX, Zhang TS, et al (2014). Abiraterone for treatment of metastatic castration-resistant prostate cancer: a systematic review and meta-analysis. Asian Pac J Cancer Prev, 15, 1313-20. 